\title{
How Agfa implemented a DAM solution
}

\section{Dirk Stevens}

studied photographics in Antwerp, Belgium. He began his professional career at Heida International, a company specialising in large format photographic printing. He joined Agfa in 1989, where he was responsible for the professional photographic lab. In 1999 he took responsibility for digital asset management (DAM) at Agfa. He is also involved with application and data integration projects.

Keywords: DAM, multi classification, Agfa, ADAM
Dirk Stevens Agfa $\mathrm{HO}$ Building 221, 4th floor Septestraat 27 B-2640 Mortsel, Belgium Tel: +32 (0)3 4448868 Fax: +32 (0)3 4005456 Email:

dirk.stevens@agfa.com

\begin{abstract}
After working for many years with a DAM solution, Agfa has set-up 12 sites, each with their own approach concerning content (read documents), functionality and users. None of them can be strictly divided, so by working with one core application, which allows the sharing of these documents without any problem across the sites, we serve our users by providing the document in the place most suitable for them and we serve our content and class administrator with central record maintenance and multi site publishing. This paper gives an overview of our multi functional DAM approach.
\end{abstract}

Around 1993 we began the move from photography to digital photography at Agfa. The first orders we received at Agfa's professional photolab were for press kits where we not only had to provide images of our products on slides and as photographic prints, but also as digital files on CD. We even briefly printed the digital images on the slide, but after a short while we only made CDs. From that moment we began digital asset management (DAM), without having any idea about the word $D A M$.

Because the photolab always took care to classify all original material from photo shoots, we had built up some knowledge in archiving hardcopy material with an index stored in a database. At that time, however, we faced the problems of having worldwide production plants for our products but
90 per cent of the marketing defined from our headquarters in Belgium. As such, all the original slides and negatives needed to be transported to HQ, where there were, and always will be, people who are only "happy" when they have the material at their desk, so sharing is only possible when they are at their desk.

Subsequently, having moved from press kit orders to posters for exhibitions and product prints with text, the logical step was to combined product prints. As a result, hardly any prints are now made directly from a color negative on photographic paper. This is a difficult reality for those who have worked for years in darkrooms, looking for that feeling of detail in the shadow areas or the fine nuance into white zones, avoiding a burnout effect on the print.

Our first DAM system was software which was very well known in the press 
world: a client-server solution, with local area network, modem and dial-in possibility. The next step was a webbased update, but limited to direct querying, direct download for lowresolution files and basket functionality for ordering a CD later on, also via FTP.

After a few years and many customizations with a lot of scripts, we made the decision of starting all over. Now we had some idea of what DAM could be and what it should be for Agfa: a web-based solution which allowed us to store all kind of file formats, could handle multilanguage for search and products, with an enhanced user policy where in-depth security granted decentralized maintenance; an open communication interface with other legacy systems, running on a standard operating system.

The vendor of our first solution had similar questions from other clients and after searching the market decided to build their solution, which turned out to be an application where we can now handle the webmaster and administrator responsibilities on a part-time basis.

There always has been, and still is, a growth path for our DAM system, which over time, has not always been the application's logical growth, depending mostly on global company decisions, but finally each functional aspect will be in put in place.

There are two main functional aspects (but in practice three): how to get data quickly and easily into the system, and how to make it as convenient as possible to retrieve these centralized data with various types of entries. As a third option: how to explore our application as much as possible. For the first two aspects I have, or I hope, some experience, however, these days many people are better trained than I.

Of course, we would like to be able to have assets which can be retrieved by searching with keywords or with "guided" searches, where relationships between assets are maintained on a semior fully automated base. These records must be indexed in as many desired languages as wanted, must hold as many documents or files as needed, whether for different color versions, different resolutions, languages, an individual page of a Quark file with respective linked files, all or only the last modified files for a production workflow document tracking solution, or any other criteria. Beside all file-related issues, we also want that only those users who have the proper profile, related to their function and business group, have access to those records reserved for them. This is solved at our company with daily maintenance where we match our users' profiles against an export file from our HR-SAP source. Based upon several criteria, users can be moved to and from specific user groups with specific content and functional rights. Based upon the same information each new user (profile) is created at the user database, where single sign on (SSO) is a prime issue.

At a multinational company, with global production facilities, it is very hard to have assets maintained centrally. With a local/wide area network and worldwide intranet, however, it is very helpful to work with decentralized content and classification administrators with specific functional application rights and centrally stored assets. The global responsibility is mostly based upon the fact that the application correctly captures all provided files and 
that content/class administrators will provide the final metadata classification. These captures can be enhanced with full-indexing, creating of additional files such as medium resolution, Windows Office compliant files, perfect cut presented previews (eg based upon Photoshop path) using dummy or other images files for formats (eg zip) without preview and other customized solutions. The archiving can be prepared locally whereby whole file directories are used for classification building and additional text files or IPTC-XML structured information for metadata capture.

The scope of the DAM application means that it is more than just an asset management system. Most of the time, DAM events such as workshops and symposia show DAM applications strictly as asset management systems only.

At Agfa we made several sub-sites in our DAM application for various reasons, with functionality of the application superseding the asset itself in some cases. Most of these sites are accessible via the intranet and the internet and have SSO access control. Users can switch between sites depending on their user group access and have specific access and functional rights to control records which are managed by the user group settings. Eighty per cent of these are built on single source code and each site has a configuration file. The sites have their own mail and notification set-up which is editable via XML, but are all running on the same application server and all data are stored in one database.

- Agfa Publishing Library (APL) was the start of our DAM solution. This site is accessible to all internal and external users, and mostly contains marketing oriented records about our products, low- to high-resolution files, PDFs and collect for print containers. The files are verified for technical aspects before publishing, such as trigger for update of publications, maintenance of father-son relationship between records, and first and second shots. Besides the standard upload routine, there is also a quick archive routine, running through an FTP module, mostly used by our press staff. APL also controls the image published at the press site and for this reason there must be a possibility to publish files at the press site, whereby the files are directly stored at APL and published on the press site, and the requester retrieves the FTP link by mail.

- The decoration site, or Deco, stores all the "nice" images that people use for decorating offices, meeting rooms, entry halls, etc. People can order highresolution files for local printing or can order images, ready for use, printed and/ or mounted on board or framed. There is a validation routine whereby the local budget responsible has to approve the order, once this is done, a mail is sent to the administration concerning order follow-up, a mail to the production house for printing and a mail to the shipment department for delivery.

- ImageBank is Agfa's own store for buying "protected" images. Basically we cover three types of restrictions: "below the line" for images which can be used for a specific purpose free from charge; "above the line" for images which can only be used after approval and/or negotiating a price for use; and finally "above the line direct" for images which are used by Agfa for one reason or another, but for which the rights have only been bought for that specific purpose - thus, in the event of wanting 
to use them again, further negotiation is required with the "external" owner. The system will inform the requester and the owner and will only release the highresolution file following the owner's approval. This functionality is enhanced with order routine for scan, because we also have a lot of useful material, available on slides (sheet format) but these are initially only scanned at a low resolution. The slides are stored at various places worldwide, but each order is placed at the correct scan production house. The files are uploaded to an FTP server, automatically stored into the database and delivered to the requester. The complete functionality can also be used at the APL site.

- Portrait is the section where we keep the portrait pictures of our employees worldwide. This site has strongly restricted access to avoid uncontrolled usage of these images. Almost every country has one or more employees responsible for upload. Using our global employee ID, they can upload the file, but in case there is no match, they still can upload and the administrator is informed about the non- verified ID upload. The ID data are retrieved from our HR-SAP, and information concerning actual status of the employee is verified on a nightly basis to ensure they are always archived in the right division/country, etc. All uploads of a particular ID are stored as multidocuments whereby only the most recent is shared with other systems, which can make direct requests or get access to a shared environment. Depending on availability, we differentiate between white background portrait images (for official purposes) or grey background images for internal usage.
Users have access from each site to their profile page, where they can modify their address and password, they also have the option whether to allow the usage of their portrait image in other applications.

- Technische Beeld Databank (TBD) or Technical Image Database is the section of the database which was created to reduce the load on the fileserver from divisions concerning image files. The concept is quite simple: mirror the actual directory structure of a fileserver under an additional root "images," inform all employees that any image files posted under that root will become available in three file formats and retrievable on a website within ten minutes. Many of these image files are used in PowerPoint or Word, but only the happy few have the required skills to reduce the file size in a proper way. By using the TBD site, users can use the preview for PowerPoint, or have the option to download a mid-resolution or the original file. The rights are again controlled by the HR-SAP structure, but as the next step we are planning to capture user rights to the active directory, so it will become a more file directory controlled site. The user can freely, from a predefined level, create directories which are directly related to the website classification structure, and become fully indexed. Even so, we index iptc and exif information and the user has the possibility to upload keywords with the use of a simple text document.

- Screens: we needed a solution for easy and fast communication - this gave us the impetus to develop another sub-site on our DAM application. Groups dedicated to communication can create 
template-driven PowerPoint slides via the web interface, set the transition and sorting, and define start and stop time. They can easily download existing slides to modify and upload again, so they become available for everyone, and this was the goal - to share slides so those responsible for communication from different divisions can use "general" information slides. Sometimes they don't really have anything new to say, in which case they use information from other groups, mostly from service divisions such as human resources or corporate communications to fill the screens. Everyone has access to all the records but they can only select those destination screens for which they are responsible. The order agent not only creates the defined presentation, but will also add the correct header text, which is different for each division or group.

- NRM, or Agfa's eBay. Active and nonactive employees from Agfa HQ can buy, bid or subscribe on waiting list for material from the factory which is no longer needed, such as chairs, desks, transport vehicles, cans, etc. Depending on certain criteria, the items are for direct sales or in case only a few of the same kind are available, we like to give everyone a fair chance, so they can place a bid via the site. Each user can only place one bid for an item. In case we have retentive items but in low quantities each time, people can subscribe to a list on the site: once they have subscribed they are locked for that item, but as soon as they have retrieved the item, they can subscribe again. The website, user maintenance and locking are done entirely through the DAM systems, but the "warehouse manipulation" is done in a Lotus Notes application, so we needed to make a "communication link" between them.
- MarCom orders. Order the right publication, as hard copy, via a DAM application. Why? Marketing material mostly has an active way of living with regard to updates. APL is the master source for storing the correct version as PDF and printable files, and has a wellstructured user policy. So instead of building a solution into an enterprise resource planning (ERP) system, we decided to enhance the DAM functionality. All local orders at Agfa need to be approved by HQ. The order may be for a short digital run or a higher volume on offset printing, or a combination of both; as such, you must be able to act fast and be sure you have the latest version at your fingertips.

Depending on the specific order, all relevant departments are directly informed.

- Docmaker light. How would you feel when you visited your company's booth at a fair and saw that your company logo was incorrectly displayed on posters, or an outdated version was being used? Even though you had sent over the CD with all correct templates and product information to the person responsible for organizing the event. Our DAM application stores all templates, users can trans-create the masters online, retrieve a low-resolution PDF by mail within ten minutes, and after approval, a link from where they can download a high-resolution PDF for local printing, or can request HQ to print the poster and send it over. The HQ panel responsible is informed each time someone approves a local version, and can even decide to make a master of that language version, so other similar native users can have a ready-to-use poster. The next generation of DocMaker, will be able to handle a six-page leaflet. 
- Business cards. Working on the same principle as DocMaker, but based upon company policy the metadata are prepared in a Notes application, and the DTP work is done by the DAM application in the background.
In my opinion, digital asset management has many faces and the return on investment will be more than foreseen, because users will guide you to their needs and you will have a key to provide the solution. 